\title{
Dynamical evolution of high velocity clouds in the intergalactic medium
}

\author{
C. Konz ${ }^{1,2}$, C. Brüns ${ }^{3,4}$, and G. T. Birk ${ }^{1,2}$ \\ 1 Institut für Astronomie und Astrophysik der Universität München, Scheinerstraße 1, 81679 München, Germany \\ 2 Centre of Interdisciplinary Plasma Science, Garching, Germany \\ 3 Radioastronomisches Institut der Universität Bonn, Auf dem Hügel 71, 53121 Bonn, Germany \\ 4 The Australia Telescope National Facility, CSIRO, PO Box 76, Epping NSW 1710, Australia
}

Received 5 February 2002 / Accepted 6 June 2002

\begin{abstract}
H}_{\mathrm{I}}$ observations of high-velocity clouds (HVCs) indicate, that they are interacting with their ambient medium. Even clouds located in the very outer Galactic halo or the intergalactic space seem to interact with their ambient medium. In this paper, we investigate the dynamical evolution of high velocity neutral gas clouds moving through a hot magnetized ambient plasma by means of two-dimensional magnetohydrodynamic plasma-neutral gas simulations. This situation is representative for the fast moving dense neutral gas cloudlets in the Magellanic Stream as well as for high velocity clouds in general. The question on the dynamical and thermal stabilization of a cold dense neutral cloud in a hot thin ambient halo plasma is numerically investigated. The simulations show the formation of a comet-like head-tail structure combined with a magnetic barrier of increased field strength which exerts a stabilizing pressure on the cloud and hinders hot plasma from diffusing into the cloud. The simulations can explain both the survival times in the intergalactic medium and the existence of head-tail high velocity clouds.
\end{abstract}

Key words. magnetohydrodynamics (MHD) - galaxies: halos - intergalactic medium - Magellanic Clouds galaxies: magnetic fields

\section{Introduction}

High-velocity clouds (HVCs) - first discovered by Muller et al. (1963) - are defined as neutral atomic hydrogen clouds with radial velocities that cannot be explained by simple galactic rotation models. After almost 40 years of eager investigations there is still no general consensus on the origin and the basic physical parameters of HVCs (e.g. Bregman 1999). The most critical issue of HVC research is the distance uncertainty. Danly et al. (1993), Keenan et al. (1995) and Ryans et al. (1997) consistently determined an upper distance limit of $d \leq 5 \mathrm{kpc}$ to HVC complex M. The most important step forward is the very recently determined distance bracket of $4 \leq d \leq 10 \mathrm{kpc}$ towards HVC complex A by van Woerden et al. (1999). These results clearly place the HVC complexes M and A in the gaseous halo of the Milky Way.

Parallel to the growing evidence that a significant fraction of the HVC complexes are located in the Milky Way halo, Blitz et al. (1999) supported the hypothesis that some HVCs are of extragalactic origin. They argued, that it is reasonable to assume that primordial gas - left over from the formation of the Local Group galaxies - may appear as HVCs. Braun \& Burton (1999) identified 65 compact and isolated HVCs and argued that this ensemble represents a homogeneous

Send offprint requests to: C. Konz,

e-mail: konz@usm.uni-muenchen.de subsample of HVCs at extragalactic distances. Observational evidence for extragalactic HVCs may also be found by the detection of the highly ionized high-velocity gas clouds by Sembach et al. (1999), because of its very low pressure of about $p k^{-1} \approx 5 \mathrm{~K} \mathrm{~cm}^{-3}$.

The Magellanic Stream (MS) and the Leading Arm (LA) (Putman et al. 1998) both form coherent structures over several tens of degrees having radial velocities in the HVC regime. They represent debris most likely caused by the tidal interaction of the Magellanic Clouds with the Milky Way. The distances to these features are of the order of $50 \mathrm{kpc}$.

Meyerdierks (1991) detected a HVC that appears like a cometary shaped cloud with a central core and an asymmetric envelope of warm neutral atomic hydrogen (the particular HVC is denoted in literature as HVC A2). He interpreted this head-tail structure as the result of an interaction between the HVC and normal galactic gas at lower velocities. Towards the HVC complex C, Pietz et al. (1996) discovered the socalled HI "velocity bridges" which seem to connect the HVCs with the normal rotating interstellar medium. The most straight forward interpretation for the existence of such structures is to assume that a fraction of the HVC gas was stripped off the main condensation. Brüns et al. (2000) extended the investigations of Meyerdierks (1991) and Pietz et al. (1996) over the entire sky covered by the new Leiden/Dwingeloo Hi 21-cm line survey (Hartmann \& Burton 1997) and found head-tail structures in all 
HVC-complexes including the Magellanic Stream, except for the very faint HVC-complex L. Their analysis revealed that the absolute value of the radial velocity of the tail is always lower than the value for the head of the HVC $\left(\left|v_{\text {LSR,tail }}\right|<\left|v_{\text {LSR,head }}\right|\right)$. In addition, it was shown that the fraction of HVCs showing a head-tail structure increases proportional to the peak column density and increasing radial velocity $\left|v_{\mathrm{GSR}}\right|$.

HVCs mostly appear as "pure" neutral atomic hydrogen clouds. Absorption line studies provide information on the ionization state and the metalicity of HVCs. The results indicate that the bright and very extended HVC complexes consist (at least partly) of processed material, having $\leq 1 / 3$ of the solar abundances (Wakker 2001).

Thus, the question of the dynamical and thermal stabilization of a cold dense neutral cloud in a hot thin ambient plasma arises. To confine a HVC in the Magellanic Stream by pressure a relatively high halo gas density is required (Mirabel et al. 1979; Weiner \& Williams 1996). Therefore, the lifetime of the HVCs should be significantly limited by evaporation (Murali 2000) since the necessary pressure for confinement is associated with a high energy transfer. An alternative mechanism of dynamical stabilization is the magnetic confinement of the cloud. In this contribution, we numerically investigate the formation of a magnetic barrier around the HVC in the Magellanic Stream and its stabilizing effects on the neutral gas cloud.

\section{Observations}

The gaseous arms of the Magellanic System are very interesting objects to study the evolution of clouds moving with high velocities relative to an ambient medium. The Magellanic Stream (MS) is a coherent structure which starts from the Magellanic Clouds and trails over $100^{\circ}$ passing the southern Galactic pole. The physical parameters of both the gas in the Magellanic Stream and the ambient medium must support survival times of the order $10^{9} \mathrm{yr}$ predicted by numerical simulations (Gardiner 1999).

The low distance of about $50 \mathrm{kpc}$ enables studies of the distribution of gas on scales of about $200 \mathrm{pc}$ with single dish telescopes. Recently, the Parkes multi-beam narrow-band survey of the entire Magellanic System was completed (see Brüns et al. 2000). Figure 1 shows exemplary the column density distribution of a part of the MS, also known as MS IV. The Stream is subdivided in two filaments containing a number of condensations. Some of them show a horseshoe like structure. In addition, the Stream is accompanied by a number of isolated clouds at similar velocities that are most likely part of the gaseous feature. The detailed analysis of the gas within the MS is difficult, as some clouds are superposed on the same line of sight. The analysis of the isolated clouds is much easier in this respect. Observable quantities are the angular extent, the line-widths, and the column density distribution. Other important values like the gaseous mass, the density etc. can only be calculated, if the distance to the clouds is known. We assume a distance of $50 \mathrm{kpc}$ to all clouds in this section.

Typical line-widths derived from isolated clouds are $F W H M \approx 25-30 \mathrm{~km} \mathrm{~s}^{-1}$, typical diameters are of the order $40^{\prime}$.

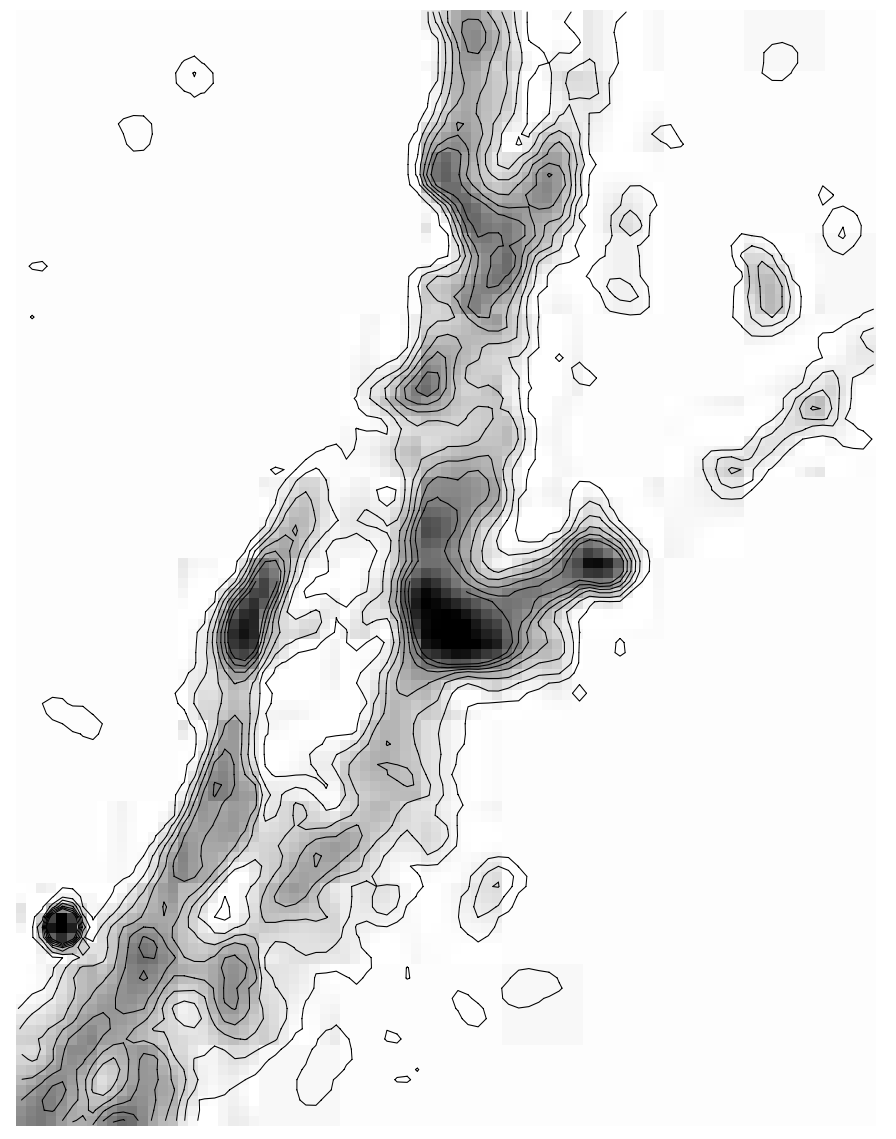

Fig. 1. This figure shows the column density distribution of a part of the Magellanic Stream observed with the multi-beam facility of the Parkes telescope. The contour levels represent $0.1,1,2,3$, ... $\times 10^{19} \mathrm{~cm}^{-2}$.

This corresponds to $580 \mathrm{pc}$ at a distance of $50 \mathrm{kpc}$. A particle moving with a velocity of $v \approx 15 \mathrm{~km} \mathrm{~s}^{-1} \approx 15 \mathrm{kpc} \mathrm{Gyr}^{-1}$ crosses the cloud in $\approx 0.04 \mathrm{Gyr}$, i.e. the crossing time is much smaller than the age of the Magellanic Stream. The virial mass of a cloud can be calculated using $M_{\mathrm{vir}}=210 r(\Delta v)^{2}$, where $r$ is the radius of the cloud in pc and $\Delta v$ is the linewidth in $\mathrm{km} \mathrm{s}^{-1}$. Virial masses for clouds in the MS are $M_{\mathrm{vir}}=3.94 \times 10^{7}$ $(r / 300 \mathrm{pc})\left(\Delta v / 25 \mathrm{~km} \mathrm{~s}^{-1}\right)^{2} M_{\odot}$, while observed HI masses are of the order few $\times 10^{4} M_{\odot}$. Apparently, the observed HI masses are orders of magnitude to low to provide gravitational stabilization. Consequently, the cloud must be confined by external pressure. Direct observations of the ambient medium provide only weak constraints on the physical parameters. The density of the ambient medium is of the order $n_{\mathrm{IGM}} \approx 10^{-5} \mathrm{~cm}^{-3}$ (Murali 2000; Weiner \& Williams 1996; Mirabel et al. 1979). The temperature of the gas in the outer Galactic halo is expected to be similar to the temperature in the lower Galactic halo $\left(T_{\mathrm{IGM}} \approx 1-2 \times 10^{6} \mathrm{~K}\right)$. For a fully ionized medium, the pressure can be calculated using $P_{\mathrm{IGM}} \mathrm{k}^{-1}=2.3 n_{\mathrm{IGM}} T_{\mathrm{IGM}}$, i.e. $P_{\mathrm{IGM} \mathrm{k}} \mathrm{k}^{-1} \approx 50 \mathrm{~K} \mathrm{~cm}^{-3}$.

The pressure within the MS can be estimated using the correlation between linewidth, turbulent velocity and kinetic temperature

$\Delta v=2\left(\ln 2\left(\frac{2 k T_{\mathrm{k}}}{m_{\mathrm{H}}}+V^{2}\right)\right)^{1 / 2}$ 


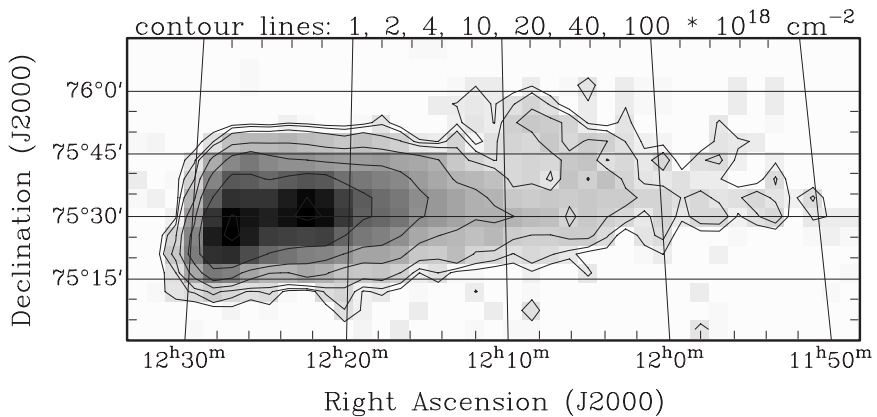

Fig. 2. This figure shows the column density distribution of HVC125+41-207. Contour lines represent 1, 2, 4, 10, 20, 40, $100 \times 10^{18} \mathrm{~cm}^{-2}$. This clouds shows a very prominent head-tail structure.

where $\Delta v$ is the linewidth, $T_{\mathrm{k}}$ is the kinetic temperature, $k$ is the Boltzmann constant, $m_{\mathrm{H}}$ is the mass of the hydrogen atom, and $V$ is the most probable turbulent velocity. The assumption that there is no turbulence provides a possibility to derive an upper limit to the kinetic temperature (a.k.a. Doppler temperature) from Eq. (1) $T_{\mathrm{D}}=21.8(\Delta v)^{2} \approx 2 \times 10^{4} \mathrm{~K}$, where $T_{\mathrm{D}}$ is in $\mathrm{K}$ and $\Delta v$ is $F W H M$ in $\mathrm{km} \mathrm{s}^{-1}$. The actual kinetic temperature must be lower, as processes like turbulence are likely to occur. Typical observed volume densities within the Magellanic Stream are of the order $n=$ few $\times 10^{-2} \mathrm{~cm}^{-3}$, assuming a distance of $50 \mathrm{kpc}$ for the Magellanic Stream. Using $P k^{-1}=n T$ allows to estimate the pressure in the Stream to be of the order $P k^{-1} \approx$ few $\times 100 \mathrm{Kcm}^{-3}$.

Pressure equilibrium between the neutral gas in the clouds and the ionized medium is possible, as both pressures are only rough estimates. In addition, the contribution of magnetic fields to the question of stability is completely unknown.

There are a number of clouds in the Magellanic Stream and the Leading Arm showing up with head-tail structures, i.e. asymmetries in the column density distribution associated with velocity gradients (Brüns et al. 2000). A detailed analysis of these features is extremely complicated, because of the superposition of clouds along the same line of sight.

Another interesting class of HVCs are the so-called compact, isolated HVCs. Braun \& Burton (1999) argue, that these HVCs are located within the intergalactic medium of the Local Group. They are by definition compact and isolated - a detailed analysis of the HI distribution is therefore much easier compared with the Magellanic Stream. Figure 2 shows the column density distribution of a compact and isolated high-velocity cloud with a distinct head-tail structure. This cloud was discussed in detail in Brüns et al. (2001). The distance to this cloud was determined to be of the order $130 \mathrm{kpc}$, i.e. the cloud is located in the very outer halo of the Milky Way or the near intergalactic space. We consider this cloud as a generic example for the class of head-tail HVCs investigated in this contribution.

Figure 3 shows the result from a Gaussian decomposition of the HI data of HVC125+41-207. About $12.5 \%$ of the HI-gas is located in the tail. The spectra show in general two components, one with a low and one with a high velocity dispersion (i.e. a cold and a warm gas-phase). The velocity dispersion of the warm HI-gas is increasing from $F W H M \approx 10 \mathrm{~km} \mathrm{~s}^{-1}$ to
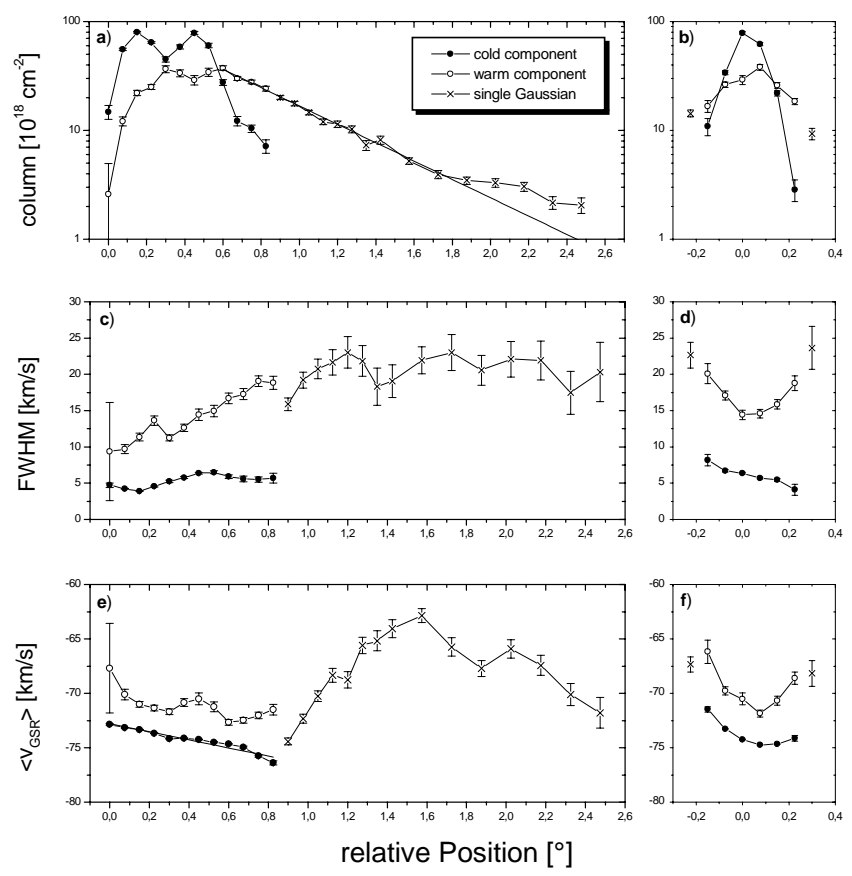

Fig. 3. This figure shows the result from a Gaussian decomposition of the HI data of HVC125+41-207 (Brüns et al. 2001). The cold gasphase is represented by filled circles, the warm gas-phase by open circles. Results from a single Gaussian fit are represented by crosses. The diagrams a, c) and e) show the results for a slice along the tail, the diagrams $\mathbf{b}, \mathbf{d}$ ) and $\mathbf{f}$ ) represent the slice perpendicular to the orientation of the tail. The line in diagram a) is an exponential fit to the data. The line in diagram e) is a fit to the velocity field.

$F W H M \approx 20 \mathrm{~km} \mathrm{~s}^{-1}$ over the extend of the cloud and remains approximately constant in the tail. This corresponds to a Doppler temperature increasing from $T_{\mathrm{D}}=2000 \mathrm{~K}$ to $T_{\mathrm{D}}=10000 \mathrm{~K}$ in the tail. There is also a trend of higher velocity dispersions towards the outskirts of the cloud. In addition, the radial velocities $\left|v_{\mathrm{GSR}}\right|$ of the warm component are lower than the velocities in the colder gas. This is a strong indication that the warm gas is in the process of being stripped off the main body of the HVC. The observed morphology of HVC125+41-207 clearly demonstrates, that this cloud is currently interacting with its ambient medium similar to the HVCs in the Magellanic Stream and Leading Arm.

Brüns (in prep.) performed deep HI observations of a sample of 10 compact HVCs with the 100-m Effelsberg telescope. All of them show up with a head-tail structure - some of the tails have a fairly low column density $\left(\approx 10^{18} \mathrm{~cm}^{-2}\right)$.

Altogether, the observations demonstrate, that HVCs in the Galactic halo or in the near intergalactic space interact with their ambient medium producing head-tail structures.

\section{Numerical simulations of high velocity clouds}

In order to study the dynamical evolution of a fast moving, cool, dense neutral gas cloud in a magnetized, hot ambient medium we performed 2-D MHD plasma-neutral gas simulations assuming invariance in the $z$-direction $(\partial / \partial z=0)$. 

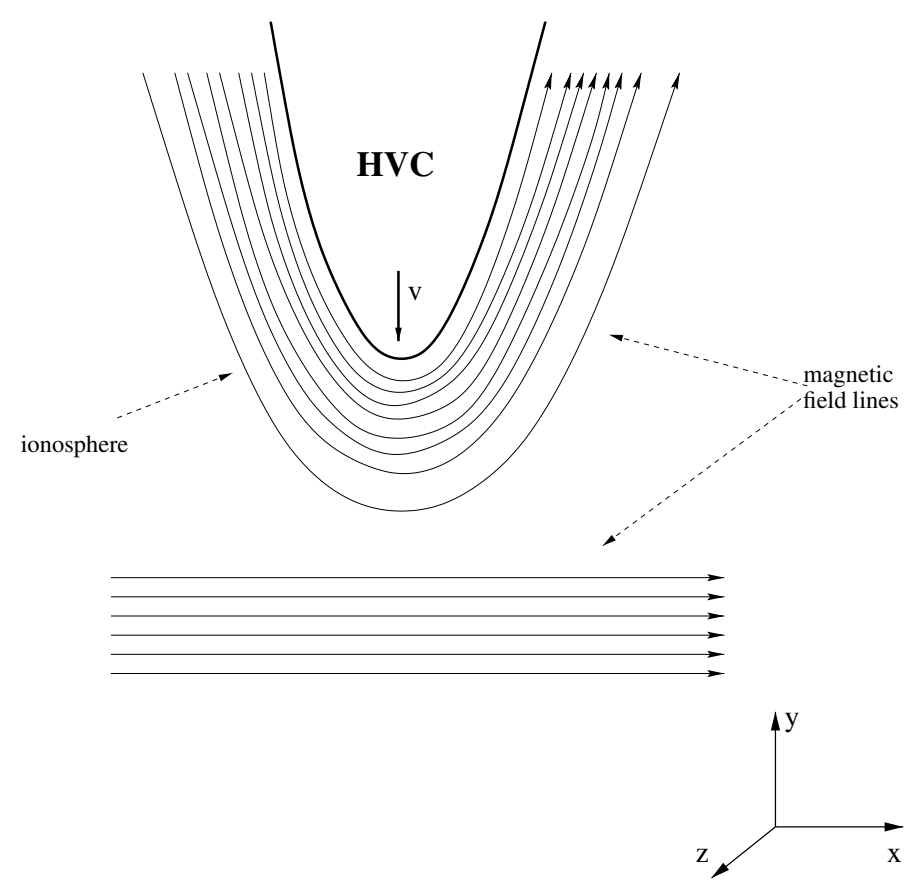

Fig. 4. A high velocity cloud moving through a magnetized plasma.

In the simulations, a dense neutral gas cloud is moving in the $y$-direction impinging on a homogeneous magnetic field lying in the $x$-direction (see Fig. 4).

Here, we consider, for example, physical parameters for HVCs in the Magellanic Stream, i.e. the outer Galactic halo. For the high velocity clouds MSI-VI in the Magellanic Stream the particle density of the ambient plasma lies in the range of $10^{-5} \mathrm{~cm}^{-3}-7 \times 10^{-4} \mathrm{~cm}^{-3}$ (Weiner \& Williams 1996; Mirabel et al. 1979) for the Galactic halo and is even lower - at about $10^{-6} \mathrm{~cm}^{-3}-$ in the intergalactic medium. The temporal evolution of the plasma-neutral gas system is calculated by numerically integrating the following set of normalized fluid equations (for a derivation of the fluid equations see Birk \& Otto 1996):

$$
\frac{\partial \rho}{\partial t}=-\nabla \cdot(\rho \boldsymbol{v})
$$

(plasma continuity equation)

$$
\frac{\partial \rho_{\mathrm{n}}}{\partial t}=-\boldsymbol{\nabla} \cdot\left(\rho_{\mathrm{n}} \boldsymbol{v}_{\mathrm{n}}\right)
$$

(neutral gas continuity equation)

$$
\begin{aligned}
\frac{\partial}{\partial t}(\rho \boldsymbol{v})= & -\boldsymbol{\nabla} \cdot(\rho \boldsymbol{v} \boldsymbol{v})-\frac{1}{2} \boldsymbol{\nabla} p+(\boldsymbol{\nabla} \times \boldsymbol{B}) \times \boldsymbol{B} \\
& -\rho v_{12}^{\mathrm{S}}\left(\boldsymbol{v}-\boldsymbol{v}_{\mathbf{n}}\right)
\end{aligned}
$$

(plasma momentum equation)

$$
\frac{\partial}{\partial t}\left(\rho_{\mathrm{n}} \boldsymbol{v}_{\mathrm{n}}\right)=-\boldsymbol{\nabla} \cdot\left(\rho_{\mathrm{n}} \boldsymbol{v}_{\mathrm{n}} \boldsymbol{v}_{\mathrm{n}}\right)-\frac{1}{2} \boldsymbol{\nabla} p_{\mathrm{n}}-\rho_{\mathrm{n}} v_{21}^{\mathrm{S}}\left(\boldsymbol{v}_{\mathrm{n}}-\boldsymbol{v}\right)
$$

(neutral gas momentum equation)

$$
\frac{\partial \boldsymbol{B}}{\partial t}=\boldsymbol{\nabla} \times(\boldsymbol{v} \times \boldsymbol{B})
$$

(induction equation)

$$
\frac{\partial p}{\partial t}=-\boldsymbol{v} \cdot \boldsymbol{\nabla} p-\gamma p \boldsymbol{\nabla} \cdot \boldsymbol{v}-3(\gamma-1) v_{12}^{\mathrm{S}}\left(p-\frac{\rho}{\rho_{\mathrm{n}}} p_{\mathrm{n}}\right)
$$

(plasma pressure equation)

$$
\begin{aligned}
\frac{\partial p_{\mathrm{n}}}{\partial t}= & -\boldsymbol{v}_{\mathrm{n}} \cdot \boldsymbol{\nabla} p_{\mathrm{n}}-\gamma_{\mathrm{n}} p_{\mathrm{n}} \boldsymbol{\nabla} \cdot \boldsymbol{v}_{\mathrm{n}} \\
& -3\left(\gamma_{\mathrm{n}}-1\right) v_{21}^{\mathrm{S}}\left(p_{\mathrm{n}}-\frac{\rho_{\mathrm{n}}}{\rho} p\right)
\end{aligned}
$$

(neutral gas pressure equation).

The quantities $\rho, p, \boldsymbol{v}, \rho_{\mathrm{n}}, p_{\mathrm{n}}, \boldsymbol{v}_{\mathrm{n}}, \boldsymbol{B}, e, \gamma$, and $\gamma_{\mathrm{n}}$ denote the plasma mass density, pressure, and velocity, the neutral gas mass density, pressure, and velocity, the magnetic field, the elementary charge, and the ratio of specific heats for the plasma and the neutral gas, respectively. The term $v v$ represents the dyadic product $\left(v_{i} v_{j}\right)_{i, j}$. The ratio of specific heats has been chosen as $\gamma=\gamma_{\mathrm{n}}=5 / 3$.

In the presented simulations, we assume a continuous ionization equilibrium. So far, we are not interested in ionization phenomena, but in the dynamical evolution of the high velocity cloud, only.

The frequencies $v_{12}^{\mathrm{S}}$ and $v_{21}^{\mathrm{S}}$ denote effective elastic collision frequencies. In order to guarantee momentum conservation, they have to fulfil the following relation (for details see Birk \& Otto 1996):

$v_{12}^{\mathrm{S}} \rho=v_{21}^{\mathrm{S}} \rho_{\mathrm{n}}$.

The symmetric collision frequency $v_{12}^{\mathrm{S}}$ is a measure for the relative collisional friction between the plasma and the neutral gas. Heuristically, we chose the reasonable ansatz

$v_{12}^{\mathrm{S}}=v_{0}^{\mathrm{S}} \rho_{\mathrm{n}} T^{1 / 2}$

for the collision frequency $v_{12}^{\mathrm{S}}$ where $T=p / \rho$ is the normalized plasma temperature and $v_{0}^{\mathrm{S}}$ is a constant factor.

All quantities $X$ are normalized to typical values $\hat{X}$ of the system. Here we chose parameters typical for high velocity clouds in the Magellanic Stream and neutral gas clouds in the intergalactic medium. Length scales are normalized to a typical extension of a cloud of $\hat{L}=100 \mathrm{pc} \approx 3 \times 10^{20} \mathrm{~cm}$. The particle density (ions and neutral atoms, respectively) is normalized to a value of $\hat{n}=10^{-3} \mathrm{~cm}^{-3}$. The magnetic field is normalized to $\hat{B}=3 \mu \mathrm{G}$. The actual magnetic field in the Galactic halo is not known. Upper limits from cosmic ray confinement, pulsars, etc. indicate field strengths of some microGauss (Vallée 1990; Beck et al. 1994). We perform the simulation with an initial field strength of one percent of the normalization field. Our findings show that even with this relatively weak initial field a substantial dynamical stabilization of the HVC results. We assume a monoatomic neutral gas, e.g. hydrogen atoms, such that the ion mass and the neutral atom mass are equal. Furthermore, we assume only one species of single ionized ions. 
One may note that a pure hydrodynamic treatment is barely applicable to HVCs in the Galactic halo since the fluid approximation does not strictly hold. With the above choice for $\hat{L}$, $\hat{n}$, and $\hat{B}$ further normalizations follow in a generic way. The mass densities $\rho$ and $\rho_{\mathrm{n}}$ are normalized to $\hat{\rho}=\left(m_{\mathrm{e}}+m_{\mathrm{i}}\right) \hat{n} \approx$ $m_{\mathrm{i}} \hat{n}=m_{\mathrm{a}} \hat{n}$ where $m_{\mathrm{e}}, m_{\mathrm{i}}$, and $m_{\mathrm{a}}$ are the electron, ion, and neutral atom mass, respectively. Velocities are normalized to the Alfvén velocity $v_{\mathrm{A}}=\hat{B} / \sqrt{4 \pi \hat{\rho}}$, frequencies to the inverse Alfvén time $1 / \tau_{\mathrm{A}}=v_{\mathrm{A}} / \hat{L}$, and pressures to $\hat{p}=\hat{B}^{2} / 8 \pi$. From the normalized equation of state for ideal gases $p=n T$ the temperature of reference can be deduced as $\hat{T}=\hat{B}^{2} / 16 \pi \hat{n} k_{\mathrm{B}}$ where $k_{\mathrm{B}}$ is the Boltzmann constant.

For the above values of the system we find a mass density $\hat{\rho} \approx 1.67 \times 10^{-27} \mathrm{~g} \mathrm{~cm}^{-3}$, the Alfvén velocity $v_{\mathrm{A}} \approx 210 \mathrm{~km} \mathrm{~s}^{-1}$, the Alfvén transit time $\tau_{\mathrm{A}} \approx 1.31 \times 10^{13} \mathrm{~s} \approx 416000 \mathrm{yr}$, and the temperature $\hat{T} \approx 1.3 \times 10^{6} \mathrm{~K}$.

For numerical reasons, the density gradient of the modeled $\mathrm{HVC}$ is chosen to be smaller than a realistic one. Consequently, the neutral gas temperature in the neutral cloud is unrealistically high. However, the dynamics are not significantly influenced by this choice since the mechanical forces are due rather to pressure than to density or temperature gradients. On the whole, we model the neutral gas as if its ionization energy was high enough to keep it neutral at $10^{6} \mathrm{~K}$. The ionization energy does not enter the calculation at any point, therefore being an arbitrary choice.

The integration of the plasma-neutral gas equations is done on an equidistant 2-D grid by a second order leapfrog scheme where the partial derivatives are realized as finite differences by the FTCS method (Forward Time Centered Space). The continuity, momentum and pressure equations are integrated with a flux correction of the source terms between the two half time steps while the induction equation is integrated by means of a semi-implicit algorithm (Dufort-Frankel). Details of the numerical code can be found in Birk \& Otto (1996).

A schematic drawing of the simulation is given in Fig. 4. In order to simulate the infall of a compact dense neutral gas cloud perpendicular to a magnetic field in a hot plasma we first transform to the neutral gas rest frame. Then the initial configuration is defined by a dense neutral gas cloud centered at the origin $(x, y)=(0,0)$ of the system having the form

$\rho_{\mathrm{n}}(r)=\rho_{\min }+\frac{\rho_{\mathrm{n}_{0}}}{\cosh a r}$.

Here, $r$ gives the distance from the origin of the system while $a=1 / 2$ defines the scale length for the neutral gas density gradient. The constants $\rho_{\min }$ and $\rho_{\mathrm{n}_{0}}$ define the minimum and the maximum neutral gas density and are chosen to be $\rho_{\min }=1$ and $\rho_{\mathrm{n}_{0}}=25$, respectively. The initial neutral gas density is shown in Fig. 5. The neutral gas cloud is initially at rest, i.e., $\boldsymbol{v}_{\mathbf{n}}=0$. The neutral gas is assumed to be initially in pressure equilibrium at a homogeneous pressure $p_{\mathrm{n}_{0}}=T_{\mathrm{n}_{0}}\left(\rho_{\min }+\rho_{\mathrm{n}_{0}}\right)$ where $T_{\mathrm{n}_{0}}=1$ is the minimum temperature of the neutral gas cloud. The temperature profile is then given by $T_{\mathrm{n}}(r)=p_{\mathrm{n}_{0}} / \rho_{\mathrm{n}}(r)$ (see Fig. 6). The neutral gas outside the cloud is about a factor 25 hotter than at the center of the cloud. This temperature gradient, however, is still small compared to realistic temperature gradients by factors of $10^{3}-10^{4}$ in typical HVCs. This is due

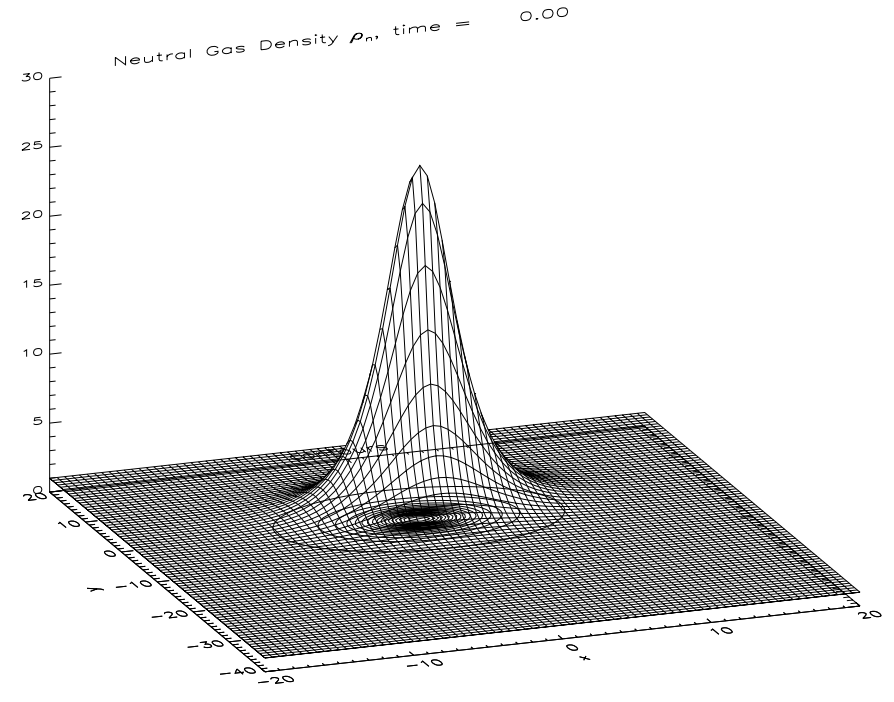

Fig. 5. The initial neutral gas density $\rho_{\mathrm{n}}$; figures are in nondimensional units.

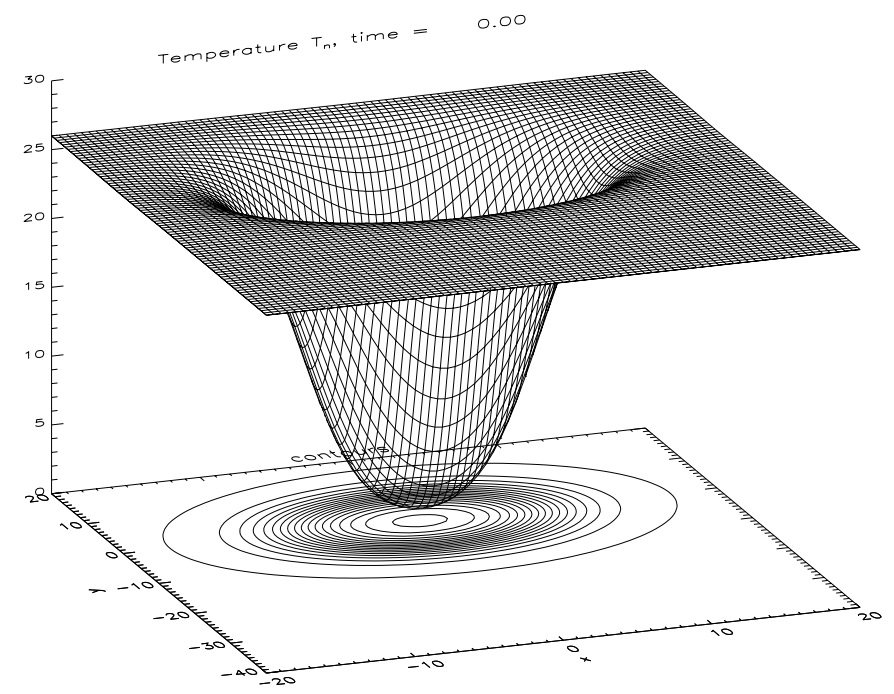

Fig. 6. The initial temperature profile $T_{\mathrm{n}}$ for the neutral gas.

to numerical reasons in so far as stronger gradients require an enhanced spatial and temporal resolution which makes a simulation extremely time- and memory-consuming. However, simulations with steeper gradients in the temperature and density of the neutral gas component have shown qualitatively the same evolution of the system apart from the longer calculation time. Since we are interested in the longterm evolution of a plasmaneutral gas system we restrain ourselves to intermediate temperature and density gradients.

The plasma itself initially has a homogeneous density $\rho_{0}=1$ and a similar temperature profile as the neutral gas:

$T(r)=T_{0}-\frac{T_{0}-T_{\mathrm{n}_{0}}}{\cosh a r}$

Here, $T_{0}=10$ is the maximum temperature of the plasma. It is worthwhile mentioning that the external neutral gas is even hotter than the external plasma in this simulation. The neutral gas outside the cloud just serves as a reservoir of heat for the outer plasma which is heated up in the course of time via 


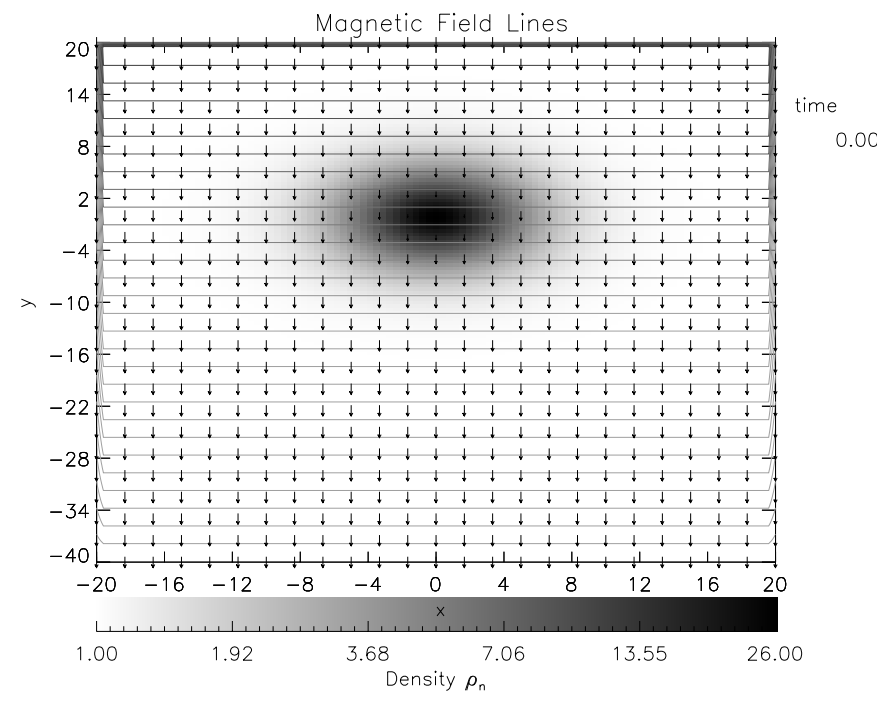

Fig. 7. The initial configuration for the neutral gas density, the plasma flow, and the magnetic field.

the collisional $v_{12}^{\mathrm{S}}$-term in the pressure equation. It is not dense enough to influence the motion of the plasma significantly due to the small value of the collision frequency $v_{0}^{\mathrm{S}}=8 \times 10^{-3}$. During the dynamical evolution, the neutral gas gathers the same velocity profile as the plasma motion. Initially, the plasma streams in the $y$-direction with the velocity profile

$v_{y}(r)=v_{y_{0}}\left(1-\frac{1}{\cosh a r}\right)$

where $v_{y 0}=-0.1$ is the minimum velocity of the plasma. This leaves the center of the neutral cloud at rest while plasma is streaming past the neutral cloud in the negative $y$-direction and across an initially homogeneous magnetic field in the $x$ direction: $B_{x}=B_{x 0}=0.01, B_{y}=B_{z}=0$. Since the local Alfvén velocity is given by $v_{\mathrm{A}}=B / \sqrt{4 \pi \rho}$ this plasma flow is superalfvénic at the beginning of the simulation due to the small magnetic field. This means that the plasma flow is able to compress and deform the magnetic field significantly. However, there is no shock to be found because of the high temperature $T \approx 1.3 \times 10^{7} \mathrm{~K}$ and, connected with it, the high velocity of sound $c_{\mathrm{s}}=\sqrt{2 \gamma k_{\mathrm{B}} T / m_{\mathrm{i}}} \approx 661 \mathrm{~km} \mathrm{~s}^{-1}$.

The complete initial configuration is shown in Fig. 7. The neutral gas density is shown in logarithmic scaling while the lines indicate magnetic field lines and the arrows indicate the plasma flow.

At the boundaries of the simulation box all quantities are extrapolated to the first order of the Taylor expansion. There are three exceptions, though. The plasma and neutral gas densities and the collision frequency $v_{12}^{\mathrm{S}}$ are chosen to be symmetric at both $y$-boundaries. Furthermore, the magnetic field component $B_{x}$ is chosen to be symmetric at the $y_{\max }$-boundary because of numerical stability reasons. The most important exception, however, is given by the fact, that the plasma flow is maintained at the upper $y$-boundary by setting $v_{y}\left(y_{\max }\right)=v_{y 0}$. Altogether, we get a plasma-neutral gas system where magnetic flux, plasma, neutral gas, and energy can freely cross the

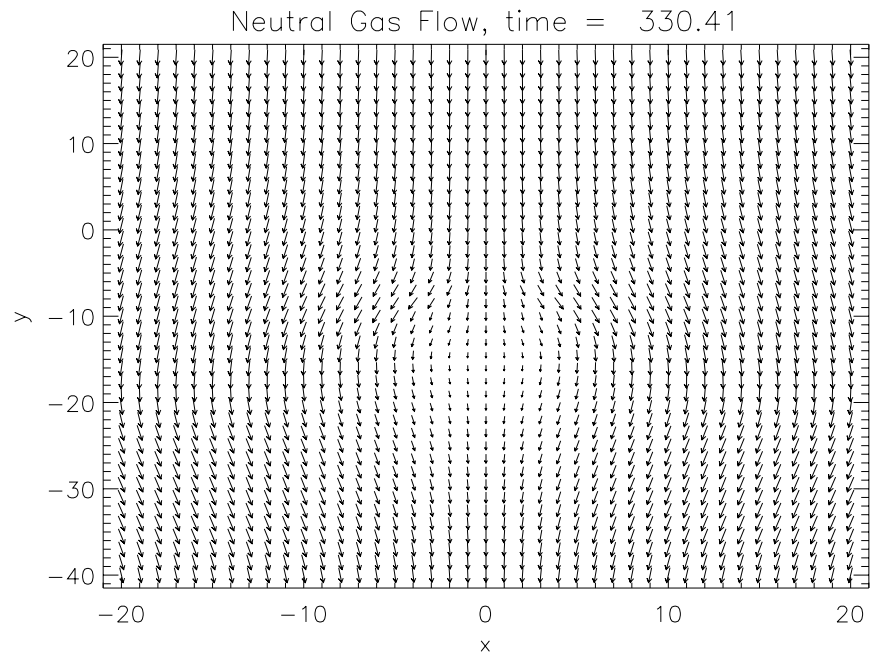

Fig. 8. The neutral gas flow at $t \approx 330 \tau_{\mathrm{A}}$.

$x$-boundaries but where magnetic flux and mass flux are partly fixed at the $y$-boundaries.

The simulations presented here are all ideal in the sense that no resistivity apart from a small constant background resistivity $\eta_{0}=10^{-5}$ is applied due to numerical reasons.

The calculations are done in a 2-D box with $x$ going from -20 to 20 and $y$ going from -40 to 20 . We use a uniform grid with 103 grid points in the $x$ - and 153 grid points in the $y$-direction resulting in a constant grid spacing of 0.4 .

Since the system is treated ideally in a magnetohydrodynamic view the magnetic field lines are frozen into the plasma. Thus, they are deformed in the course of time by the plasma streaming past the neutral gas cloud. At the beginning of the simulation, both the neutral gas and the plasma at the origin are at rest. The collisional terms $\rho v_{12}^{\mathrm{S}}\left(\boldsymbol{v}-\boldsymbol{v}_{\mathbf{n}}\right)$ and $\rho_{\mathrm{n}} v_{21}^{\mathrm{S}}\left(\boldsymbol{v}_{\mathbf{n}}-\boldsymbol{v}\right)$ in the momentum equations lead to a deceleration of the plasma impinging upon the neutral gas cloud and to an acceleration of the neutral gas cloud itself. However, the acceleration of the neutral gas cloud is rather small due to the high neutral gas density of the cloud. Outside the cloud, plasma and neutral gas densities are comparable such that the neutral gas acquires the same velocity as the streaming plasma.

The neutral gas flow is shown in Fig. 8. One finds a velocity pattern that resembles the initial velocity pattern apart from the fact that the center of the neutral gas cloud has moved towards the lower boundary. Furthermore, the plasma and neutral gas is now flowing around the cloud as if it was a soft obstacle in a hydrodynamic flow. The center of the neutral gas cloud is now moving with a small velocity in the negative $y$-direction as a result of the continuous impact of plasma on the cloud. The exchange of momentum between the incoming plasma and the resting neutral gas cloud is performed via elastic ion-neutral gas collisions. The momentum exchange leads to an acceleration of the cloud in the initial rest frame, i.e., to a deceleration of the cloud in the plasma rest frame. This is consistent with ultraviolet absorption observations hinting at a deceleration of the HVCs as they approach the Galactic disk (Benjamin 1999; Danly 1989). 


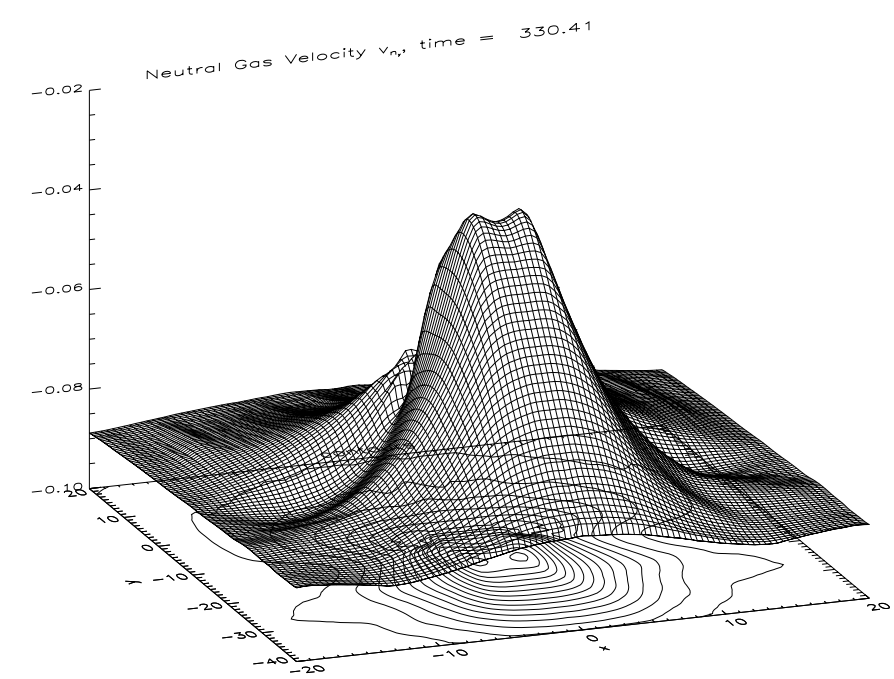

Fig. 9. The neutral gas velocity $v_{\mathrm{n}_{y}}$ at $t \approx 330 \tau_{\mathrm{A}}$.

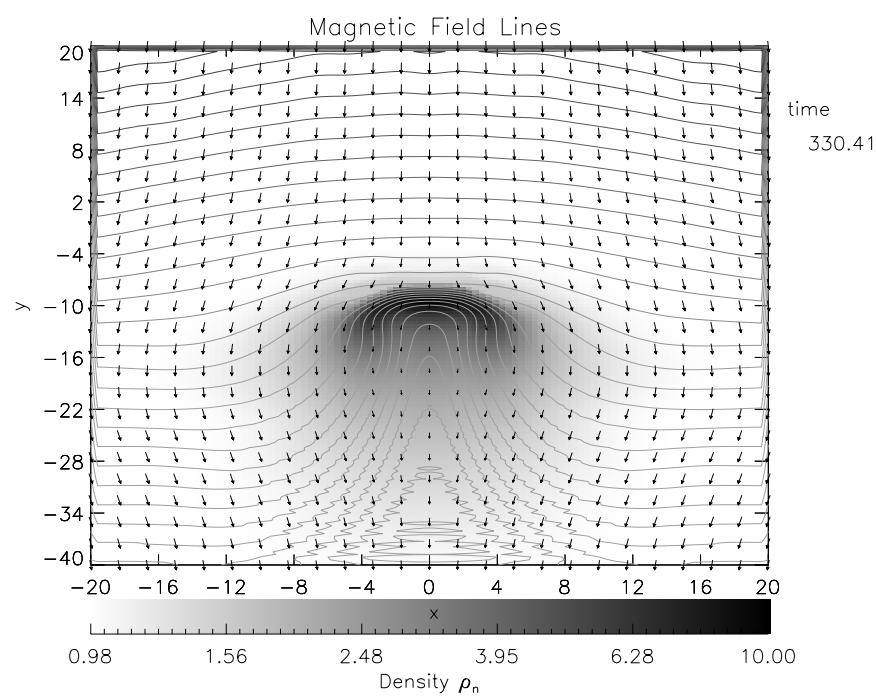

Fig. 10. The neutral gas density, the plasma flow, and the magnetic field at $t \approx 330 \tau_{\mathrm{A}}$.

Figure 9 shows the neutral gas velocity $v_{\mathrm{n}_{y}}$ at the same time as the flow in Fig. 8. As already mentioned the center of the cloud is now moving with a small but finite velocity in the negative $y$-direction.

The simulation time of about 330 Alfvén times corresponds to a time interval of about 140 million years which is comparable to crossing times of HVCs in the Magellanic Stream.

A complete representation of the system after about 330 Alfvén times is given in Fig. 10. The arrows represent the flow of the plasma around the neutral gas cloud. Since the cloud does not act as a solid but as a soft obstacle there are no real stagnation points in front and behind the cloud. Nevertheless, the plasma flow encountering the cloud is partly redirected sideways similar to a hydrodynamic stream around a solid obstacle. Since the magnetic field lines are frozen in the plasma, magnetic flux is transported via the plasma flow towards the front edge of the cloud while the field lines on both sides of the cloud are draped around the cloud forming a magnetic barrier at the front and a magnetotail at the wake of the HVC.

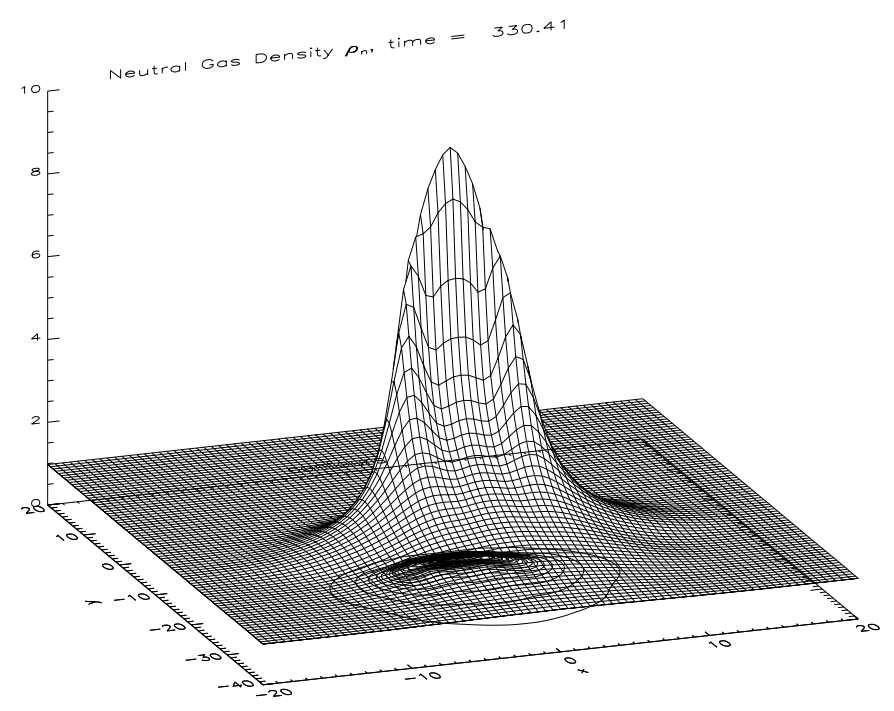

Fig. 11. The neutral gas density at $t \approx 330 \tau_{\mathrm{A}}$.

The formation of a magnetic barrier around the head of the neutral gas cloud resembles very much the formation of a magnetic barrier at the front of a HVC via the critical velocity effect (Konz et al. 2001). In turn, the formation of a magnetotail is quite similar to the formation of a magnetotail by the wellstudied interaction of the solar wind with unmagnetized planets (Luhmann 1986; Luhmann et al. 1992) and comets (Formisano et al. 1982; McComas et al. 1987; Flammer et al. 1997).

The neutral gas cloud itself is slightly deformed into a U-like structure, visible in Fig. 10 in logarithmic scaling of the neutral gas density, together with a weak tail consisting of neutral gas being slowly stripped off the wings of the cloud. The stripping of neutral gas off the edges of the cloud results from the momentum transfer from the halo gas to the neutral gas particles. The stability of the cloud is not influenced significantly by this ongoing effect during the time period under consideration. Eventually, this abrasion of neutral gas forms a tail. The phenomenon of tail formation is consistent with the observation (see Fig. 3). The upper column of this figure shows that the tail mainly consists of the warm component while the colder core of the neutral gas cloud remains unaffected by the stripping. After the formation of the magnetic barrier the resulting density profile of the cloud, as it is shown in Fig. 11, remains almost constant in time as later time-steps of the simulation demonstrate. The HVC almost keeps its width and its maximum density.

The dynamical stability of the neutral gas cloud is a direct effect of the magnetic confinement by the accumulated magnetic barrier. This can be shown by performing the same simulation without any magnetic field. Figures 12 and 13 show the neutral gas density at about $300 \tau_{\mathrm{A}}$ and $540 \tau_{\mathrm{A}}$ for this case. One finds the initially compact neutral gas cloud being first disrupted into two major parts and then completely falling apart. The cold neutral cloud is unstable against impact of hot plasma. The momentum and energy transfer from the incoming plasma to the front of the cloud leads to a disruption of the cloud into several parts. 


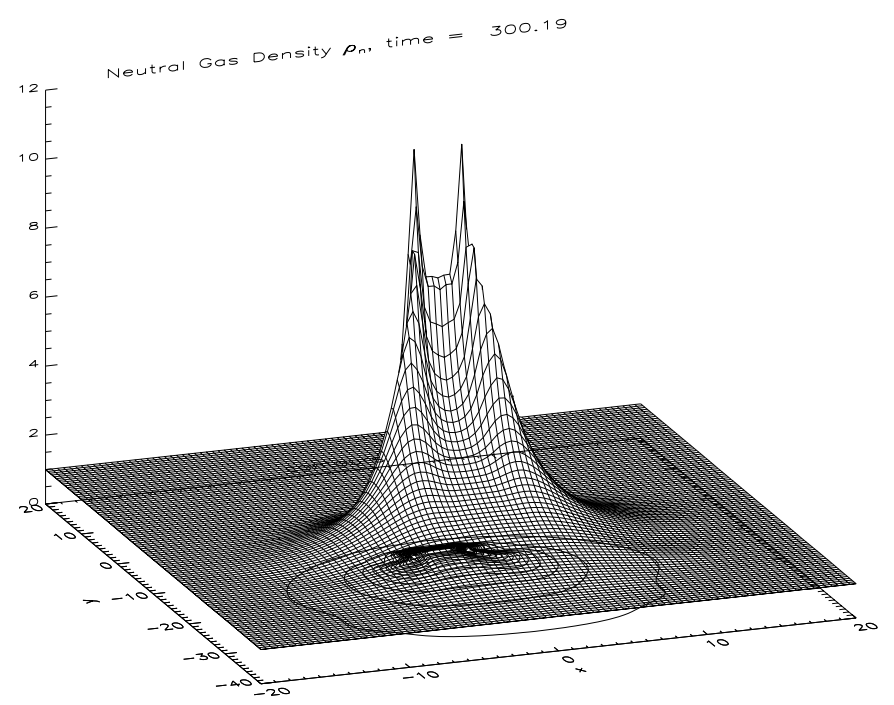

Fig. 12. The neutral gas density at $t \approx 300 \tau_{\mathrm{A}}$ for the non-magnetic case.

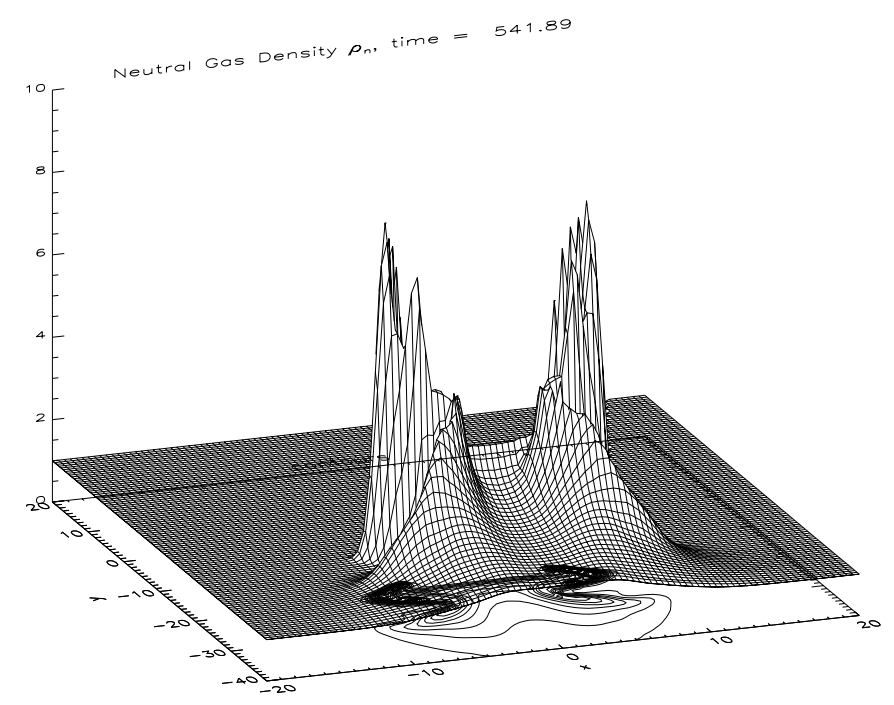

Fig. 13. The neutral gas density at $t \approx 542 \tau_{\mathrm{A}}$ for the non-magnetic case.

For comparison to the case of a magnetic field initially perpendicular to the flow direction, Figs. 14 and 15 show the cloud and the magnetic field configuration for the case of an inclined magnetic field with initial field strengths $B_{x 0}=B_{y 0}=0.03$. As in the previous simulation, the initial plasma inflow is purely in the negative $y$-direction. As long as there is a significantly strong component of the magnetic field perpendicular to the flow direction the formation of a magnetic barrier stabilizes the cloud in two ways. First of all, the increased magnetic field in the barrier exerts a magnetic pressure $\boldsymbol{B}^{2} / 8 \pi$ on the cloud and partly compensates the ram pressure $\rho \boldsymbol{v}^{2} / 2$ of the plasma stream. Since the system is ideal in the magnetohydrodynamic description plasma cannot move across magnetic field lines. Therefore, the plasma flow is redirected by the magnetic barrier to stream around the HVC. On the inner side of the magnetic barrier, the magnetic pressure is compensated by the internal plasma pressure.

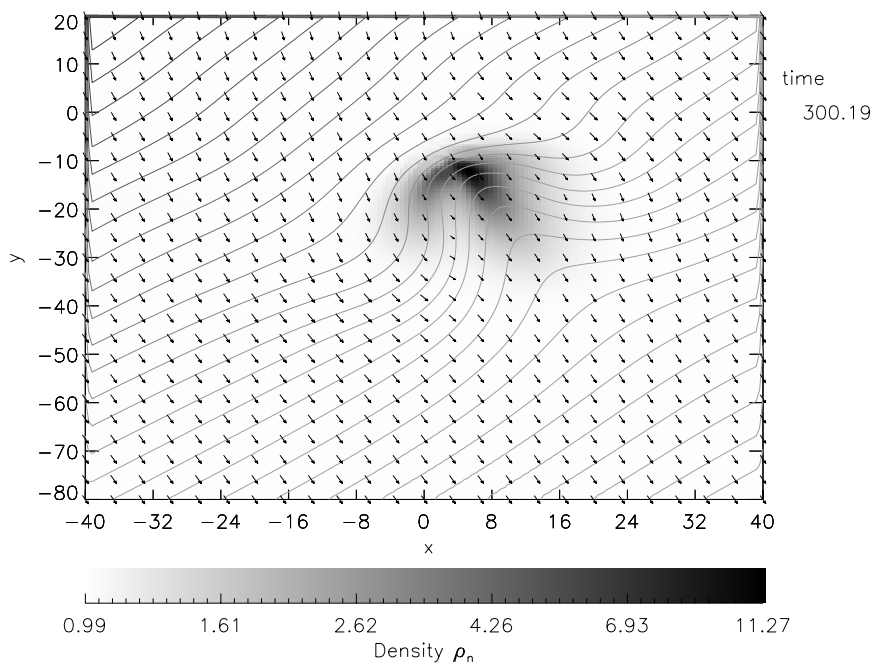

Fig. 14. The neutral gas density, the plasma flow, and the magnetic field at $t \approx 300 \tau_{\mathrm{A}}$ for the case of an inclined inflow magnetic field.

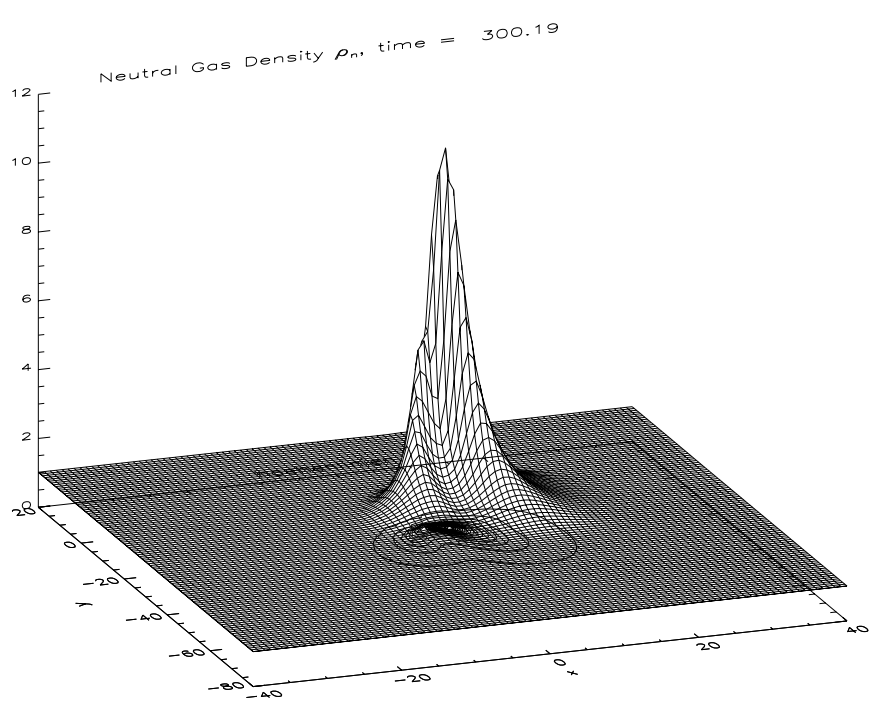

Fig. 15. The neutral gas density at $t \approx 300 \tau_{\mathrm{A}}$ for an inclined inflow magnetic field.

As expected, we find a lower limit of the magnetic field strength sufficient to stabilize the HVC. For the parameters chosen, this limit is about $15 \mathrm{nG}$ meaning one order of magnitude higher than the mean intergalactic magnetic field (Vallée 1983). At the beginning of the simulation, the plasma density was set to be homogeneous. However, there is a pressure gradient due to the fact that the plasma inside the cloud region is much cooler than outside the cloud. Due to the finite temperature of a HVC and neutral gas ionization via UV photons, collisions and soft $\mathrm{X}$-ray background there is always a small fraction of plasma inside a HVC amounting to the order of several percent of the total density. In the course of the simulation, this fraction is increased up to $50 \%$ by plasma streaming towards the center of the cloud along the field lines, i.e., along the tail and by a compression of the whole cloud due to the external plasma pressure. The plasma inside the cloud, however, is cooled by energy transfer to the cold neutral gas and would be subject to recombination events if recombination was 
included in the simulation. So far, recombination is not considered in our simulations. However, recombination of the cold plasma inside the cloud would further increase the build-up of a magnetic barrier because the plasma pressure gradient would be even steeper due to the loss of plasma. That means that more magnetic flux is transported inside the magnetic barrier and the magnetic tail by the plasma heading for the cold center of the cloud. The magnetic pressure finally stops this plasma flow and isolates the plasma inside the cloud from the ambient halo plasma. In our simulation, the plasma inside the cloud takes up to $50 \%$ of the total density. This percentage, however, only reaches such a high level because of the relatively small density of the neutral gas cloud compared to the density of the ambient plasma. For a higher neutral gas density the plasma fraction inside the cloud only amounts to a few percent.

Altogether, we have a system consisting of three different regions: an outer region of hot ambient plasma exerting the ram pressure on the region of the magnetic barrier and the inner region with cold plasma and neutral gas compensating the magnetic pressure from inside. Elastic collisions inside the cloud are much more frequent than outside since the higher neutral gas density overcompensates the lower temperature in Eq. (10). Therefore, plasma and neutral gas inside the cloud have the same temperature and the same velocity.

The magnetic barrier not only dynamically stabilizes the HVC but also thermally. The thermal conduction perpendicular to a magnetic field is drastically reduced compared to the thermal conduction parallel to the magnetic field (Braginskii 1965). The coefficient $\kappa_{\perp}$ for the heat conduction perpendicular to the magnetic field decreases with increasing magnetic field strength as $B^{-2}$ (Van der Linden \& Goossens 1991). Figure 16 shows the magnetic field strength after about $330 \tau_{\mathrm{A}}$. At the front of the HVC, the magnetic field strength is increased by a factor 10 , in the tail by a factor 4 . This means that the perpendicular heat conduction is reduced by a factor $1 / 100$ or $1 / 16$, respectively. In our simulation, heat conduction was not included so far. Since the main part of the temperature gradient is perpendicular to the magnetic field lines no qualitative changes are to be expected if heat conduction is included.

Heat conduction is suppressed and plasma cannot cross the magnetic barrier the temperature of the neutral gas cloud remains almost constant in time. Figure 17 shows the temperature profile of the neutral gas after about $330 \tau_{\mathrm{A}}$. Although a little bit deformed the initial temperature profile is almost maintained. Steep temperature gradients are found in the region of the magnetic barrier surrounding the minimum temperature at the maximum of the neutral gas density. The temperature on the outside is still by a factor 10 higher than on the inside of the cloud. The magnetic barrier therefore insulates the cloud against heat exchange and heat conduction.

In the tail, the temperature gradient is much smaller leading to a tail at an intermediate temperature. This relatively warm neutral gas in the tail shows a higher velocity dispersion than the core of the cloud itself, a feature that has been confirmed by measuring the velocity dispersion both in the head and in the tail of HVCs (see Sect. 2).

How is the neutral gas density in the simulation compared to observations of head-tail structures? Figure 18 shows a cut

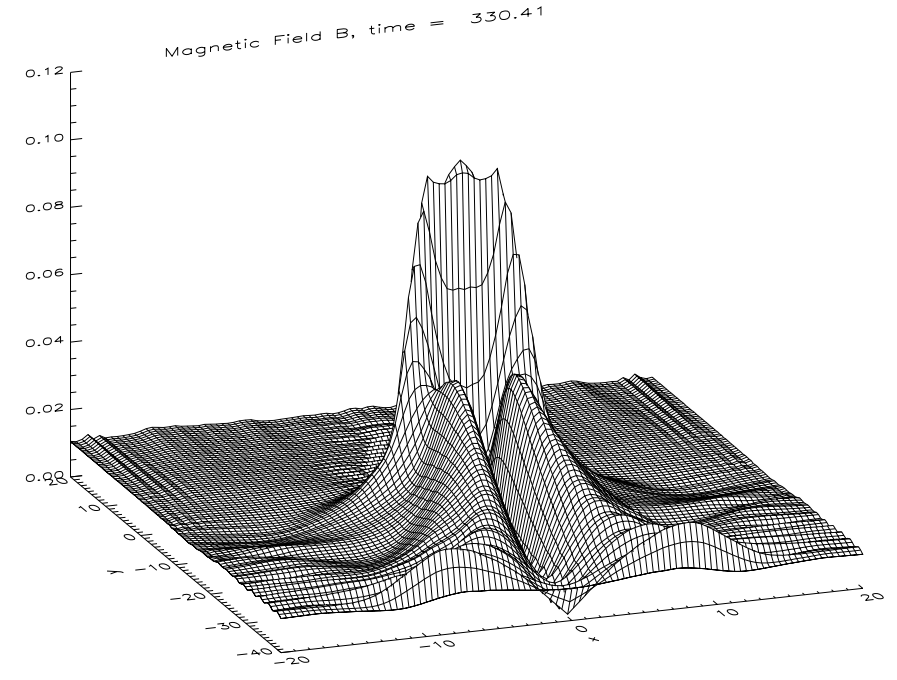

Fig. 16. The magnetic field strength at $t \approx 330 \tau_{\mathrm{A}}$.

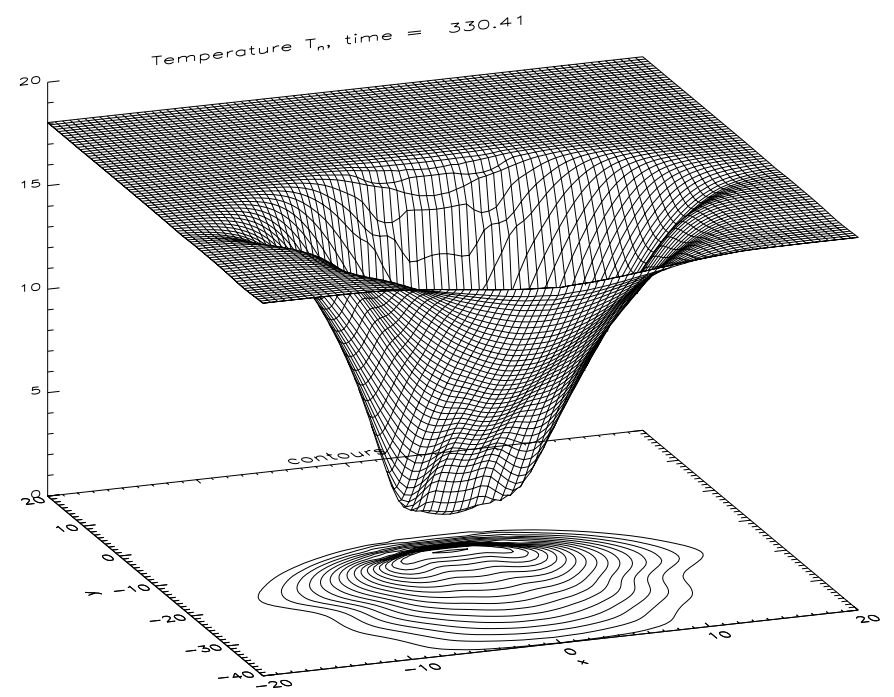

Fig. 17. The neutral gas temperature at $t \approx 330 \tau_{\mathrm{A}}$.

along the $y$-axis for the neutral gas density, i.e., along the axis of symmetry. In the plasma rest frame, the neutral gas cloud is moving to the right, i.e., in the positive $y$-direction. The density cut shows a steep gradient at the head of the cloud where the cloud is well confined by the magnetic barrier and a smooth almost exponential decay towards the tail. In this presentation, the HVC looks like a comet trailing in its wake a tail of material that is stripped off its surface in the course of time. For the HVC, the situation is quite similar. Neutral gas is slowly stripped off the outer wings of the cloud by momentum transfer between plasma and neutral gas. The stripped gas atoms or molecules form a tail-like structure with a higher temperature than the core of the cloud and a much smaller density. The tail itself is laterally shielded by magnetic field lines draped around the cloud forming a magneto-tail such that plasma and heat exchange between tail and ambient medium are reduced. The simulated HVC shows the same morphology and qualitatively similar density and temperature profiles as typical HVCs. Moreover, the velocity pattern (Fig. 9) shows the same 


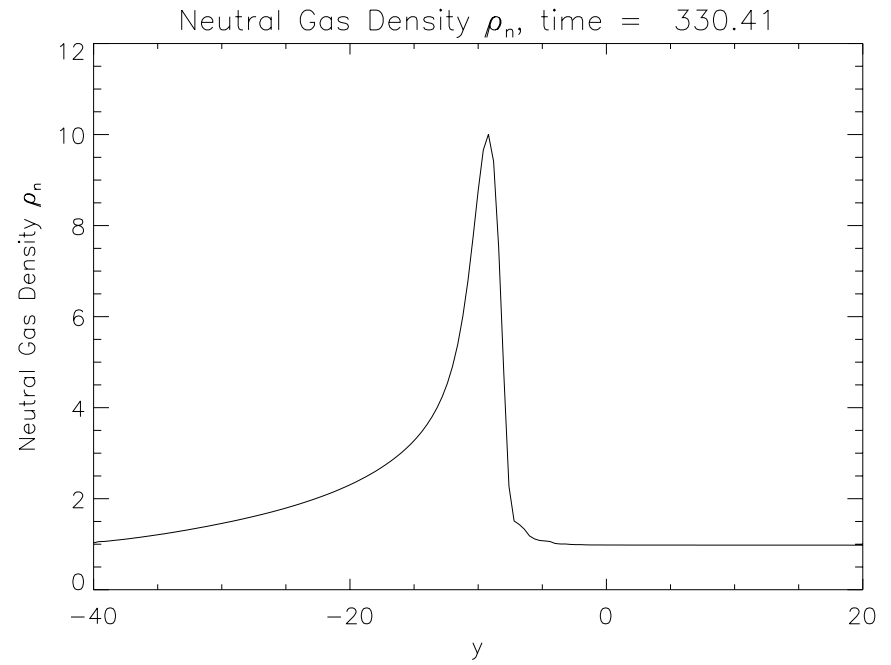

Fig. 18. A neutral gas density cut along the $y$-axis at $t \approx 330 \tau_{\mathrm{A}}$.

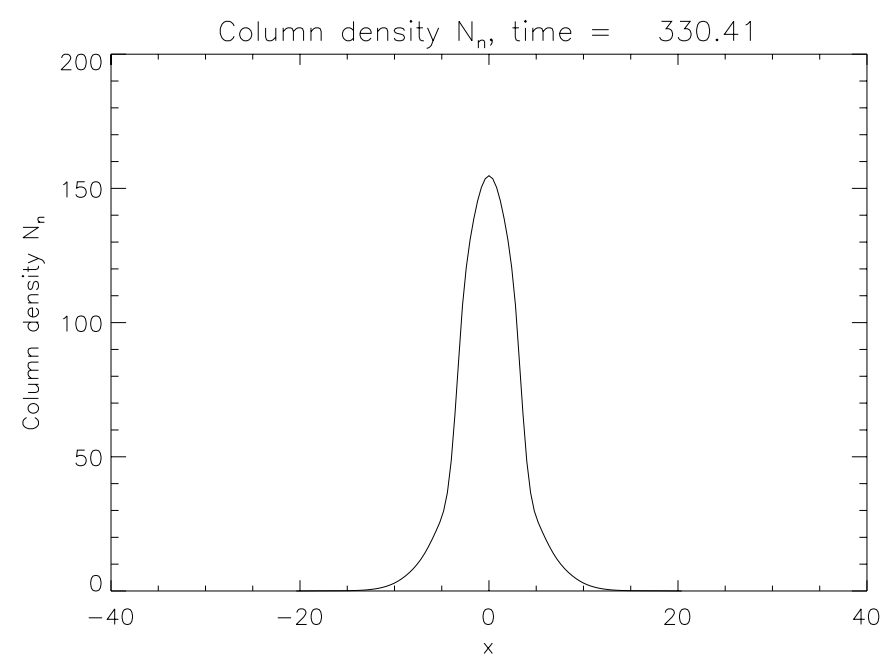

Fig. 19. A neutral gas column density cut along the $x$-axis at $t \approx$ $330 \tau_{\mathrm{A}}$.

characteristics as the observed HVC presented in Fig. 3: a fast moving cold core, slower moving warm boundary layer and a slowly moving tail.

Finally, Fig. 19 shows the column density for the neutral gas for a cut along the $x$-axis through the core of the cloud. Here, we calculated the column density by rotating the cloud around the $y$-axis and integrating along the line of sight lying in the $z$-direction. This corresponds to measuring the column density by integrating along the line of sight. Since we initially assumed invariance in the $z$-direction this is of course not exact but reasonable for clouds which are not too small. The result shows a smooth profile which is qualitatively very similar to column densities shown, for example, in Fig. 3.

All in all, the simulation shows the importance of the magnetic field for the morphology of the HVC as well as for its stability.

\section{Discussion}

We presented the first plasma-neutral gas simulations of the interaction of HVCs with the Galactic halo gas. Previous numerical studies (Santillaán 1999 and Quilis 2001) focused on the dynamics in external, Galactic gravitational fields. The questions of thermal and magnetic insulation of the neutral gas clouds in the hot ambient medium as well as their stability were not addressed. Our two-fluid approach provides rather detailed information on the interaction region of the cold almost neutral HVC gas and the hot almost fully ionized ambient plasma. The Galactic magnetic field is locally draped around the HVC, i.e., a magnetic barrier forms similar to magnetopauses around comets (McComas 1987) and unmagnetized planets (Luhman 1986). This barrier is of great importance for the thermal insulation of the cold, fast traveling cloud from the hot surroundings. Furthermore, it prevents the plasma from diffusing into the neutral gas cloud. We conclude that the formation of magnetic barriers contributes significantly to the dynamical stability of HVCs. Even for relatively weak initial magnetic fields in the Halo the magnetic barrier sufficiently stabilizes the cloud. In particular, unstable Kelvin-Helmholtz modes are not excited due to the stabilizing effect of a magnetic field component parallel to the flow. Thermal radiative losses that are not included in our contribution may further enhance the stability of the boundaries against Kelvin-Helmholtz modes (Vietri et al. 1997). Within the regions of discontinuity and in the adjacent inner layers the physics becomes rather involved. In particular, some fraction of the stored magnetic field energy can be converted into heat by magnetic reconnection and thus can be responsible for the observed x-ray emission (Zimmer et al. 1997; Birk et al. 1998). Moreover, one may note that the effect of ionization caused by the relative cloud halo movement (Konz et al. 2001) may enhance the formation of magnetic barriers by ionized boundary layers.

A self-consistent three-dimensional simulation including all relevant physical effects seems to be highly desirable and is a promising task for the future.

Finally, we note that the interaction scenario studied in this paper may also be of relevance in the context of cloudlets in the magnetospheres of Active Galactic Nuclei as well as in cluster cooling flows.

Acknowledgements. Part of this work was supported by the German Deutsche Forschungsgemeinschaft, DFG projects, LE 1039/5-1, LE 1039/6-1 and ME 745/19.

We thank the referee for his helpful comments.

\section{References}

Beck, R., Carilli, C. L., Holdaway, M. A., \& Klein, U. 1994, A\&A, 292, 409

Benjamin, A. J. 1999, in Stromlo Workshop on HighVelocity Clouds, ed. B. K. Gibson, \& M. E. Putman (San Francisco: ASP), ASP Conf. Ser., 166, 147

Birk, G. T., \& Otto, A. 1996, J. Comp. Phys. 125, 513-525

Birk, G. T., Lesch, H., \& Zimmer, F. 1998, Phys. Scr. T74, 89 
Blitz, L., Spergel, D. N., Teuben, P. J., Hartmann, D., \& Burton, W. B. 1999, ApJ, 514, 818

Blom, J. J., Bloemen, H., Bykow, A. M., et al. 1997, A\&A, 321, 288

Braginskii, S. I. 1965, Rev. Plasma Phys. 1, 205

Braun, R., \& Burton, W. B. 1999, A\&A, 341, 437

Bregman, J. N. 1999, ASP Conf. Ser., 166, 88

Brüns, C., Kerp, J., Kalberla, P. M. W., \& Mebold, U. 2000, A\&A, 357,120

Brüns, C., Kerp, J., \& Staveley-Smith, L. 2000, in Mapping the Hidden Universe: The Universe Behind the Milky Way - The Universe in H I, ed. R. C., Kraan-Korteweg, \& P. A. Henning, ASP Conf., 218, 349

Brüns, C., Kerp, J., \& Pagels, A. 2001, A\&A, 370, L30

Danly, L. 1989, ApJ, 342, 785

Danly, L., Albert, C. E., \& Kuntz, K. D. 1993, ApJ, 416, L29

Flammer, K. R., Mendis, D. A., \& Shapiro, V. D. 1997, ApJ, 482, 1021

Formisano, V., Galeev, A. A., \& Sagdeev, R. Z. 1982, Planet. Space Sci., 30, 491

Gardiner, L. T. 1999, in Stromlo Workshop on High-Velocity Clouds, ed. B. K. Gibson, \& M. E. Putman (San Francisco: ASP), ASP Conf. Ser., 166, 292

Hartmann, D., \& Burton, W. B. 1997, An Atlas of Galactic Neutral Hydrogen Emission (Cambridge University Press)

Herbstmeier, U., Mebold, U., Snowden, S. L., et al. 1995, A\&A, 298, 606

Keenan, F. P., Shaw, C. R., Bates, B., Dufton, P. L., \& Kemp, S. N. 1995, MNRAS, 272, 599

Kerp, J., Burton, W. B., Egger, R., et al. 1999, A\&A, 342, 213

Konz, C., Lesch, H., Birk, G. T., \& Wiechen, H. 2001, ApJ, 548, 249

Luhmann, J. G. 1986, Space Sci. Rev., 44, 241
Luhmann, J. G., Russel, C. T., Brace, H., \& Vaisberg, O. L. 1992, in Mars, ed. H. Kieffer, C. Snyder, \& B. Jakobsky (Tucson: Univ. Arizona Press), 1090

McComas, D. J., Gosling, J. T., Russel, C. T., \& Slavin, J. A. 1987, J. Geophys. Res., 92, 10111

Meyerdierks, H. 1991, A\&A, 251, 269

Mirabel, I. F., Cohen, R. J., \& Davies, R. D. 1979, MNRAS, 186, 433

Muller, C. A., Oort, J. H., \& Raimond, E. 1963, C.R. Acad. Sci. Paris, 257,1661

Murali, C. 2000, ApJ, 529, L81

Pietz, J., Kerp, J., Kalberla, P. M. W., et al. 1996, A\&A, 308, L37

Putman, M. E., Gibson, B. K., Staveley-Smith, L., et al. 1998, Nature, 394, 752

Quilis, V., \& Moore, B. 2001, ApJ, 555, L95

Ryans, R. S. I., Kenan, F. P., Sembach, K. R., \& Davies, R. D. 1997, MNRAS, 289, 83

Santillán, A., Franco, J., Martos, M., \& Kim, J. 1999, ApJ, 515, 657

Sembach, K. R., Savage, B. D., Lu, L., \& Murphey, E. M. 1999, ApJ, 515,108

Vallée, J. P. 1983, Astron. Lett., 23, 8

Vallée, J. P. 1990, A\&A, 239, 57

Van der Linden, R. A. M., \& Goossens, M. 1991, Sol. Phys., 134, 247

van Woerden, H., Schwarz, U. J., Peltier, R. F., Wakker, B. P., \& Kalberla, P. M. W. 1999, Nature, 400, 138

Vietri, M., Ferrara, A., \& Miniati, F. 1997, ApJ, 483, 262

Wakker, B. P., \& van Woerden, H. 1997, ARA\&A, 35, 217

Wakker, B. P. 2001, ApJS, 136, 463

Weiner, B. J., \& Williams, T. B. 1996, AJ, 111, 1156

Zimmer, F., Lesch, H., \& Birk, G. T. 1997, A\&A, 320, 746 